\title{
Iridium Implantation in T1 and T2 Cancers of Anterior Tongue: Report from B. P. Koirala Memorial Cancer Hospital, Nepal
}

\author{
Vijayakumar Narayanan $^{1 *}$, Bibek Acharya ${ }^{2}$, Chaurasia Pradumna Prasad ${ }^{2}$, Dejkumar Gautam $^{3}$ \\ ${ }^{1}$ Clinical Director, Cancer Care Kenya, Nairobi, Kenya \\ ${ }^{2}$ Department of Radiation Oncology, B. P. Koirala Memorial Cancer Hospital, Bharatpur, Nepal \\ ${ }^{3}$ Department of Otorhinolaryngology, B. P. Koirala Memorial Cancer Hospital, Bharatpur, Nepal \\ Email:*drvijay@cancercarekenya.com
}

Received July 22, 2012; revised October 7, 2012; accepted October 15, 2012

\begin{abstract}
Early stage cancers of tongue are treated traditionally with a wide local excision or hemiglossectomy, but the preservation of normal speech and swallowing are hampered. Most of the patients are treated with external beam irradiation to achieve the best locoregional control as only a limited number of tongue cancers can be excised. Underdeveloped nations with finite resources are still dependent on cobalt based external beam radiotherapy and sometimes a Linear Accelerator with two dimensional planning. This treatment has many limitations, as the large radiation fields irradiate not only the tumor but also normal tissue. The sequalae include mucositis, dry mouth, teeth and gum injury, spinal cord damage and rarely mandibular necrosis. Intensity modulated radiotherapy, which can abrogate these side effects, is not available to these patients. Irradiation using implanted solid radioactive sources into the tumor tissue is a viable option in this context. This kind of treatment is termed as brachytherapy and if the implant is introduced into the tissue then it is interstitial brachytherapy. This report details our experience in interstitial implantation, planning, dosimetry and treatment. Diagnosed cancers of anterior 2/3rd of lateral border of tongue with T1 N0M0 or T2 N0M0 stages were subjected to Iridium implantation under general anesthesia. Orthogonal films were taken and planning done with brachyvision treatment planning system. High dose rate radiotherapy was delivered as per the prescription. Excellent local control of the tumor was achieved with no undue morbidity to the adjacent structures. The patients were asked to undergo regular follow up. Surgical salvage was advised in cases of nodal recurrence. Interstitial implantation is a treatment that can be safely administered in early stage cancers of the tongue. This has remarkable efficacy and is also a patient friendly procedure.
\end{abstract}

Keywords: Oral Cavity Cancer; Interstitial Brachytherapy; Implantation; Remote after Loader

\section{Introduction}

Brachytherapy is the treatment of malignant lesion using radioactive material at a short distance or placed inside target tissue in which there is rapid dose fall outside the tumor. The idea is to produce a dose distribution tailored to the target volume with subsequent sparing of normal tissue [1]. Based on the site of treatment, it is divided into three procedures-intracavitary, interstitial and intraluminal brachytherapy. Intracavitary brachytherapy service started in September 2002 at B. P. Koirala Memorial Cancer Hospital (BPKMCH), Bharatpur, Nepal with a Varisource High Dose Rate (HDR) Remote after Loader machine with 10 Curies Iridium-192 $\left({ }^{192} \mathrm{Ir}\right)$ radioactive source. ${ }^{192} \mathrm{Ir}$ is a solid single radioisotope of 5 $\mathrm{mm}$ length; $0.348 \mathrm{~mm}$ diameter which emits gamma rays

"Corresponding author. of $380 \mathrm{keV}$ and a short half-life of 73.83 days. In the last decade, we have treated around 3200 patients, mostly gynecological cancers with intracavitary brachytherapy and few esophageal cancers with intraluminal brachytherapy. This report discusses the interstitial Iridium implantation in T1 and T2 cancers of anterior tongue, its dosimetry, planning and treatment. Though interstitial implantation is beneficial than surgical procedures, they have not been practiced in Nepal till recently due to procedural and dosimetric complexity.

\section{Materials and Method}

All patients were diagnosed cases of carcinoma anterior 2/3rd of tongue, and TNM staging was T1 NOM0 or T2 N0M0. Pre-implant care included dental status evaluation, antiseptic mouth washes and prophylactic antibiotics. The 
patients were deemed fit for general anesthesia. The target volume was identified intraoperatively by palpation and direct visualization.

Figure 1 shows a tumor of size $1 \mathrm{~cm}$ in longest diameter on the right lateral border of anterior 2/3rd of the tongue. Procedure was performed under general anesthesia through submandibular approach. Implants were placed in such way that they cover the tumor volume with at least $1 \mathrm{~cm}$ margin. Needles were placed equidistantly 1 $\mathrm{cm}$ apart from each other and catheters were introduced and secured with buttons. Nasogastric feeding tube was inserted.

Figure 2 shows the patient with guide tubes with buttons in situ after a successful implantation for a T1 tumor on the right lateral border of anterior 2/3rd of the tongue. Tubes were put $1 \mathrm{~cm}$ apart. Orthogonal films were taken with dummy wires inside the catheter in Ximatron simulator and were scanned by Vidar film digitizer. The entire pixel size was calibrated so that measurement on film produced correct magnified coordinates. Registration point was taken at the isocentre of two orthogonal images. Reference points, applicator location length, step size, first and last source position were entered in the brachyvision.

Figure $\mathbf{3}$ is a photograph from the brachyvision. It shows the lateral X-ray film of the patient with dummy wires. Reference points are marked. Brachyvision treatment planning system is part of Varisource treatment unit. The Paris system dose specification uses a basal dose rate which is the average of the dose rates calculated at each minimum dose points in the central transverse plane of the implant. It is at the mid point between wires in a single plane and at the center of the triangles or squares formed by wires in a multiple plane implant. The dose rate used for prescription is the reference dose rate which is equal to $85 \%$ of the basal dose rate [2].

Reference points are shown in Figure 4. All are 0.5 $\mathrm{cm}$ apart and the active source dwell length in each wire was $3 \mathrm{~cm}$. The basal dose value was $6.5 \mathrm{~Gy}$. The prescribed dose per fraction, i.e. $85 \%$ of basal dose, was 5 Gy. Dwell time was computed based on current source wire activity from calibration date and scale factor applied to initial planes. The isodose curve was edited and volumes of higher dose region such as $8 \mathrm{~Gy}, 10 \mathrm{~Gy}$ in different colors were compared with prescribed dose region in $3 \mathrm{D}$ dose volume as well as in color wash to make final dwelling time acceptable for the plan.

Varisource treatment unit is a Remote after Load (RAL) equipment. The radiation source, ${ }^{192} \mathrm{Ir}$ is secured in a shielded safe, to protect healthcare professionals from radiation exposure. After ascertaining the correct positioning of patient and the applicators on him/her, the other end of the applicators were connected to the "Remote After Loader" machine (containing the radioactive sources) through a series of connecting guide tubes (Figure 5). The treatment plan was sent to the Remote after Loader, which then controls the delivery of the sources along the guide tubes into the pre-specified positions within the applicator. This process is initiated only after all staff members exit the treatment room. The sources remain in place for the pre-specified length of time, following the treatment plan. Once the treatment is over, they return along the tubes to the Remote after Loader.

The total prescribed dose was 50 Gy in 10 fractions/ twice daily, i.e., $5 \mathrm{~Gy}$ in each fraction. The prescribed dose was $15 \mathrm{~Gy}$ in 3 fractions for those who were treated with external beam radiotherapy up to $60 \mathrm{~Gy}$. A practical version of isoeffect formula based on Linear Quadratic model was applied to calculate acute early effects for tumors and late effects for normal tissue based on $\alpha$ / $\beta$ with values 10 and 3 respectively [3]. A hard copy of the treatment plan and dwell time calculation details were printed and recorded and all dosimetry plan verifycation, and personal information for treatment details were transferred into a CD ROM and exported. Four applicators were used to give a prescribed dose of 5 Gy through 4 channels to complete treatment within 4 - 5 minutes.

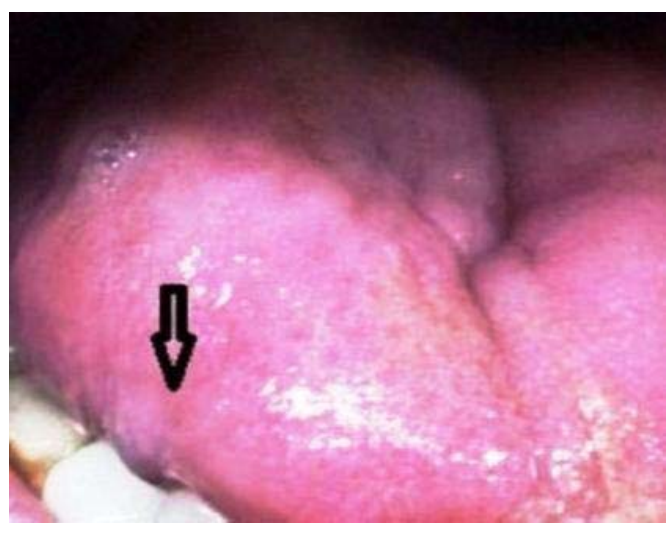

Figure 1. T1 tumor of tongue.

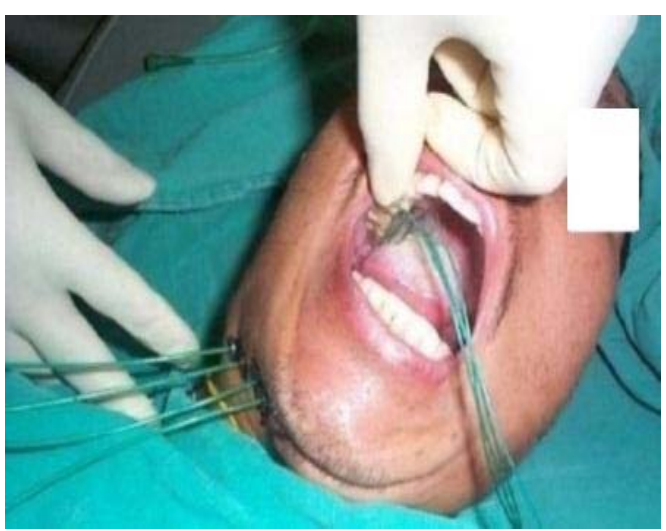

Figure 2. Implant. 


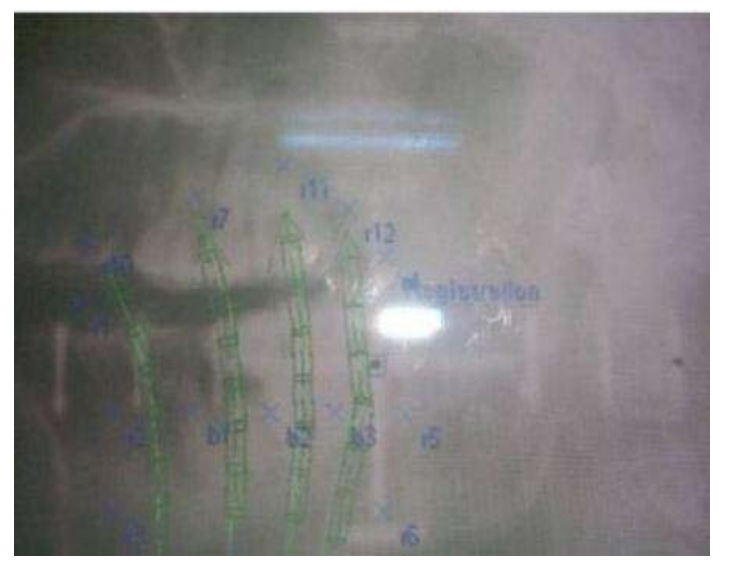

Figure 3. Lateral X-ray.

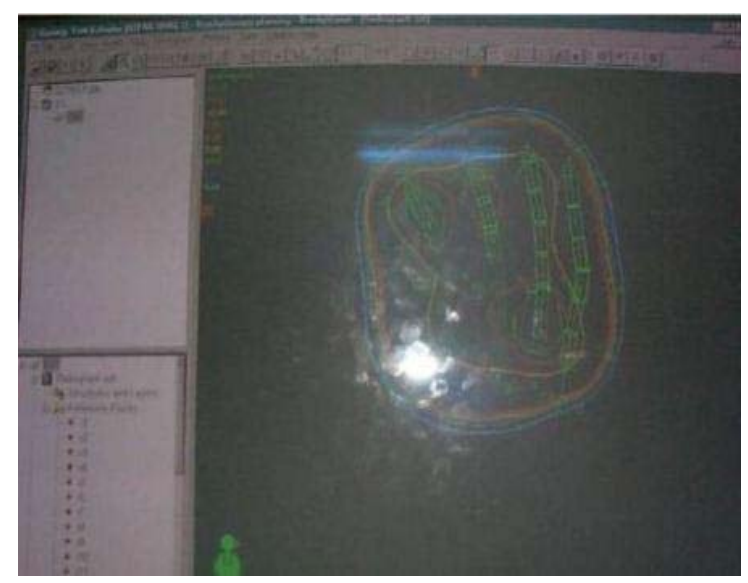

Figure 4. Isodose distribution.

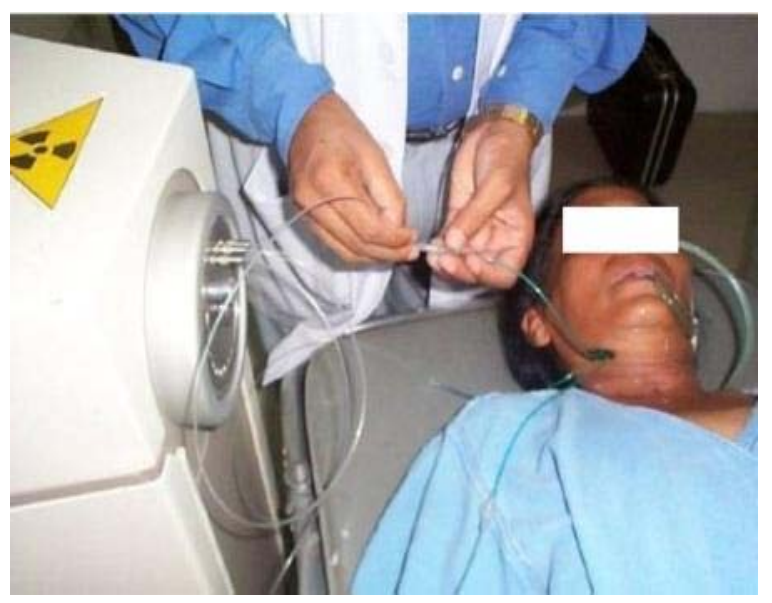

Figure 5. Connecting to Remote after Loader.

\section{Results and Discussion}

Cancer of the oral cavity constitutes $18.8 \%$ of head and neck cancers in Nepal [4], and of this cancer of tongue constitute around $30 \%$. A wide variety of treatments had been tested in the last 25 years, which include surgery, radiotherapy, concurrent chemoradiotherapy, interstitial implantation etc. [5-10], though traditionally these tumors are treated with surgery. Early lesions like T1 and $\mathrm{T} 2$ are usually excised per orally with $\mathrm{T} 1$ requiring large wedge resection and T2 with hemiglossectomy. Most patients experience functional difficulty after surgery which includes difficulty in articulation and dysphagia.

Radiation therapy is an alternative to surgery in early and intermediate size tumors of tongue. The advantage of radiation therapy in $\mathrm{T} 1$ and $\mathrm{T} 2$ tumors is its ability to provide functional and anatomical continuity. External beam radiotherapy from a teletherapy machine is the widely used method to deliver radiation. Most of the cancer treatment centers in underdeveloped countries use telecobalt machine to deliver the radiation, while few centers use Linear Accelerators.

A novel treatment option in radiotherapy is the intensity modulated radiation therapy (IMRT). It has a potential for better loco-regional control in inoperable disease due to its capability to spare the normal tissues from unwanted side effects of radiotherapy. Improved loco-regional outcome following IMRT has been reported for oropharyngeal tumors [11-14]. Underdeveloped countries like Nepal cannot afford the sophisticated therapy equipment. Logistic reasons and financial constraints guide them to conventional radiotherapy. Usage of large radiation portals in conventional radiotherapy not only irradiate the tumor but also normal tissues, resulting in many side effects including dry mouth, erythema, spinal cord injury, brachial plexopathy and mandibular necrosis. In this context, interstitial brachytherapy with its excellent results in early stage $\mathrm{T} 1$ and $\mathrm{T} 2$ tumors of the mobile tongue is a promising therapeutic option.

Experiences from treatment procedures have shown that a total dose of 60 - 70 Gy by implant alone can achieve excellent tumor control. The neck can be observed for surgical salvage in case of nodal recurrence [15].

In some centers, external radiotherapy followed by implantation is used. The argument is that in tumors more than $2 \mathrm{~cm}$ diameter, the possibility of nodal metastasis is more than 25\% [16]. Both surgery and radiotherapy has similar control for T1 and T2 tumors, approximately 80\% for T1and $60 \%$ for T2 tumors. The 5 year survival is around $70 \%$ for stage I and 50\% for stage II [17]. When iridium implantation alone is used, the local control is $87 \%$ in $\mathrm{T} 1$ and $\mathrm{T} 2$ tumors and 5 year survival was 52\% for stage I and 44\% for stage II.

The Paris method of defining the $85 \%$ of isodose rate curve relative to the minimum central dose gives consistency to implant therapy, prescribing and reporting. The differential in dose should be set before charting the treatment plan. The dose within the target volume is not homogeneous and dose to tissue in contact with source can be very high. At the periphery an isodose rate curve can be chosen for prescription. The dose rate changes by 
a factor of 2 or more over a distance of few millimeters. Correct assignment of planned catheters to treatment channels is to be checked. There is individualized planning for every patient.

For prescribing dose to HDR interstitial treatment, radiobiological aspects are considered compatible to Low dose rate (LDR) and hot spot should be in small volume compared to large treatment volume with homogeneous dose. The dwelling time in the mid applicators are lesser than the peripheral ones. For acute early effect to tumor and late effect to normal tissue, the HDR 50 Gy dose is equal to $62.5 \mathrm{~Gy}$ and $80 \mathrm{~Gy}$.

The side effects of this treatment can be grouped into acute morbidities and late effects as the most important acute morbidities are procedure related. Others are mucositis, taste alteration, transient difficulty in swallowing. These can be managed effectively with symptomatic measures. The late effects may include xerostomia resulting in dry mouth, speech and swallowing difficulty, dental caries. Stricture formation, ulceration and necrosis can also occur in late stage [18].

Apart from grade II mucositis; we did not come across any other side effects. This lasted for 2 - 3 weeks, and was resolved with symptomatic management. Excellent local control of the tumor was achieved in all cases. Patients were advised to undergo regular follow up. In case of nodal recurrence, surgical salvage was advised. All patients were found to be diseases free after 3 months post treatment. They were followed up for 5 years, and remained tumor free highlighting the efficacy of the procedure (Figure 6).

\section{Conclusion}

Interstitial brachytherapy is primarily curative and an organ preserving form of treatment. The potential advantage of the brachytherapy is that it delivers higher doses to the target volume simultaneously sparing the normal tissues, hence a conformal type of radiotherapy. This

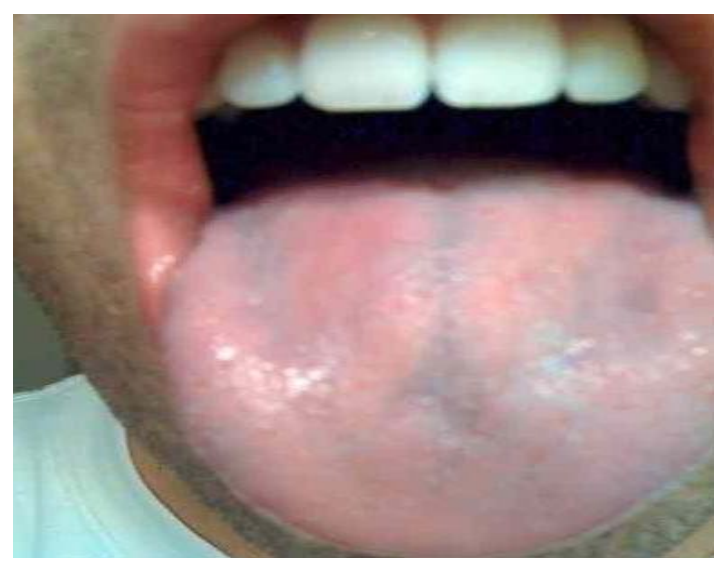

Figure 6. Normal tongue after 5 years. type of therapy can be thought of as an extreme form of hyper fractionation, killing cells by directly lethal events. Since the time interval of 6 hours was maintained between fractions, some amount of sub lethal damage repair occurs. The overall treatment time is very short, so it is very convenient to the patient. Radiation is accurately applied to tumor and hence geographical miss factor is eliminated. This is a modern and precise technique which requires concerted action.

\section{REFERENCES}

[1] M. T. Gillin, et al., "Practical Considerations for Interstitial Brachytherapy,” University of Wisconsin, Madison, 1988.

[2] S. A. Leibel, T. I. Phillips, et al., "Text Book of Radiation Oncology,” WB Saunders, Philadelphia, 1998.

[3] J. R. Williams and D. I. Thwarts, "Radiotherapy in Practice,” Oxford University Press, 1993.

[4] D. K. Baskota, R. Agrawal, et al. "Distribution of Malignancies in Head and Neck Regions and Their Management,” Journal of the Nepal Medical Association, Vol. 44, No. 159, 2005, pp. 68-72.

[5] S. W. Beenken, H. Krontiras, W. A. Maddox, G. E. Peters, S. Soong and M. M. Urist, "T1 and T2 Squamous Cell Carcinoma of the Oral Tongue: Prognostic Factors and the Role of Elective Lymph Node Dissection," Head \& Neck, Vol. 21, No. 2, 1999, pp. 124-130. doi:10.1002/(SICI)1097-0347(199903)21:2<124::AID-H ED5>3.0.CO;2-A

[6] D. G. Sessions, G. J. Spector, J. Lenox, B. Haughey, C. Chao and J. Marks, "Analysis of Treatment Results for Oral Tongue Cancer,” Laryngoscope, Vol. 112, No. 4, 2002, pp. 616-625. doi:10.1097/00005537-200204000-00005

[7] M. A. Gonzalez-Moles, F. Esteban, A. Rodriguez-Archilla, et al, "Importance of Tumour Thickness Measurement in Prognosis of Tongue Cancer,” Oral Oncology, Vol. 38, No. 4, 2002, pp. 394-397. doi:10.1016/S1368-8375(01)00081-1

[8] T. M. P. Amaral, A. R. Da Silva Freire, et al., "Predictive Factors of Occult Metastasis and Prognosis of Clinical Stages I and II Squamous Cell Carcinoma of the Tongue and Floor of the Mouth,” Oral Oncology, Vol. 40, No. 8, 2004, pp. 780-786. doi:10.1016/j.oraloncology.2003.10.009

[9] J. J. Grau, J. Domingo, J. L. Blanch, E. Verger, et al., "Multidisciplinary Approach in Advanced Cancer of the Oral Cavity: Outcome with Neoadjuvant Chemotherapy According to Intention-to-Treat Local Therapy. A Phase II Study,” Oncology, Vol. 63, No. 4, 2002, pp. 338-345. doi:10.1159/000066226

[10] R. W. Hinerman, W. M. Mendenhall, C. G. Morris, et al., "Postoperative Irradiation for Squamous Cell Carcinoma of the Oral Cavity: 35-Year Experience," Head \& Neck, Vol. 26, No. 11, 2004, pp. 984-994. doi:10.1002/hed.20091

[11] N. Lee, D. R. Puri, A. I. Blanco and K. S. Chao, "Intensity-Modulated Radiation Therapy in Head and Neck 
Cancers: An Update,” Head \& Neck, Vol. 29, No. 4, 2007, pp. 387-400. doi:10.1002/hed.20332

[12] D. R. Puri, W. Chou and N. Lee, "Intensity-Modulated Radiation Therapy in Head and Neck Cancers: Dosimetric Advantages and Update of Clinical Results," American Journal of Clinical Oncology, Vol. 28, No. 4, 2005, pp. 415-423. doi:10.1097/01.coc.0000162443.08446.00

[13] F. F. de Arruda, D. R. Puri, J. Zhung, A. Narayana, et al., "Intensity-Modulated Radiation Therapy for the Treatment of Oropharyngeal Carcinoma: The Memorial SloanKettering Cancer Center Experience,” International Journal of Radiation Oncology *Biology* Physics, Vol. 64, No. 2, 2006, pp. 363-373. doi:10.1016/j.jjrobp.2005.03.006

[14] K. S. Chao, G. Ozyigit, A. I. Blanco, W. L. Thorstad, et al., "Intensity-Modulated Radiation Therapy for Oropharyngeal Carcinoma: Impact of Tumor Volume,” International Journal of Radiation Oncology * Biology ${ }^{*}$ Physics, Vol. 59, No. 1, 2004, pp. 43-50. doi:10.1016/j.ijrobp.2003.08.004
[15] J. J. Mazzeron, J. M. Crook, V. Benck, et al., "Iridium Implantation of T1 \& T2 Carcinoma of Mobile Tongue," International Journal of Radiation Oncology "Biology Physics, Vol. 19, No. 6, 1990, pp. 1369-1376. doi:10.1016/0360-3016(90)90346-L

[16] D. Dearnaley, C. Dardaufas, et al., "Interstitial Irradiation for Carcinoma of Tongue and Floor of Mouth: Royal Marsden Hospital Experience 1970-1986,” Radiotherapy \& Oncology, Vol. 21, 1991, pp. 183-190. doi:10.1016/0167-8140(91)90036-G

[17] D. Sloan and H. Goepfert, "Conventional Therapy for Head and Neck Cancer," Hematology/Oncology Clinics of North America, Vol. 5, No. 4, 1991, pp. 601-625.

[18] M. Umeda, H. Komatsubara, N. Nishimatsu, et al., "High Dose Rate Interstitial Brachytherapy for Stage I-II Tongue Cancer," Oral Surgery, Oral Medicine, Oral Pathology, Oral Radiology and Endodontology, Vol. 90, No. 5, 2000, pp. 667-670. doi:10.1067/moe.2000.110087 